\title{
Ärztinnen und Ärzte tragen bei zur vorsorglichen Verminderung elektromagnetischer Felder im privaten und öffentlichen Raum
}

\author{
Ärztinnen und Ärzte haben eine wichtige Rolle in der gesundheitlichen Vor- \\ sorge. Die vorsorgliche Verminderung von potentiell schädlichen Umweltein- \\ flüssen (wie elektromagnetischen Feldern - EMF) gehört dazu. Die AefU be- \\ richten über eine Aktion bei den Gemeinden und Schulen zur vorsorglichen \\ Verminderung der Belastung mit EMF. Die Möglichkeiten der Einflussnahme \\ durch Ärztinnen und Ärzte werden aufgezeigt und Hilfsmittel angeboten.
}

\section{Arbeitsgruppe \\ «Elektromagnetische Felder und Gesundheit» der AefU* \\ * Dr. med. Edith Steiner-Rüedi, Dr. med. Yvonne Gilli, \\ med. pract. Cornelia Semadeni, Dr. med. Bernhard Aufdereggen}

Korrespondenz:

Dr. med. Edith Steiner-Rüedi

Friedbergstrasse 32

CH-8200 Schaffhausen
Die Belastung mit elektromagnetischen Feldern (EMF) im privaten und öffentlichen Raum nimmt laufend zu. Der Ausbau der Mobilkommunikation geht weiter. Ganze Städte und Ortschaften werden flächendeckend mit WLAN (mobiler Zugang des Computers mittels Funksignal zum Internet) ausgerüstet. In Schulen wird mittels WLAN die Verbindung zum Internet ermöglicht.

Wir Ärztinnen und Ärzte für Umweltschutz (AefU) befürchten durch die zunehmende Belastung der Bevölkerung mit elektromagnetischen Feldern gesundheitliche Risiken, v.a. auch bei Kindern. Wir erachten die vorhandenen Hinweise auf eine Beeinflussung der menschlichen Gesundheit durch Funk, Hochspannungsleitungen, Transformatoren sowie elektrische und elektronische Geräte als ausreichend, um vorsorglich tätig zu werden [1].

Ärztinnen und Ärzte haben als Entscheidungsträger in Spitälern und in der eigenen Praxis, als Schulärzte oder als Berater von Entscheidungsträgern und als engagierte Bürgerinnen und Bürger wesentlichen Einfluss auf Entscheidungen im Zusammenhang mit der Installation von Anlagen mit elektromagnetischen Feldern. Im Sinne der Vorsorge hier tätig zu werden heisst, dazu beizutragen, dass die Exposition mit EMF auf das notwendige Minimum reduziert wird. Auch in Ihrer Gemeinde gibt es elektrosensible Menschen, die unter dieser Belastung leiden. Auch Sie fragen sich, ob die Installation dieser Infrastruktur gesundheitlich problematisch ist, insbesondere in den Schulen. Sie sind direkt mit den Klagen Betroffener und den Fragen von Besorgten konfrontiert und müssen Stellung beziehen.
Die AefU haben in einer Aktion in der Deutschschweiz (geplant ist auch ein Versand in der Romandie und im Tessin) die Gemeinden und die Vorsteherinnen und Vorsteher der Schulen angeschrieben, um auch diese Stellen auf ihre Verantwortung für die Belastung des öffentlichen Raumes und der Schulen mit elektromagnetischen Feldern hinzuweisen. Wir haben dazu an die Gemeinden und Schulvorstehenden ein Merkblatt: «Elektrosmog und Gesundheit - Möglichkeiten zur Vorsorge auf Gemeindeebene» und ein Faltblatt «Gesundheit und Elektrosmog im Schulalltag» versandt.

Denn auf Gemeindeebene gibt es unter Wahrung des gesetzlichen Rahmens manche Betätigungsfelder, um vorsorglich die Belastung mit nichtionisierender Strahlung (NIS) zu reduzieren. In diesem Sinne haben wir die Behörden in den Gemeinden und Schulen $\mathrm{zu}$ folgenden Massnahmen aufgefordert:

- Information der Bevölkerung über die Gesundheitsrisiken der elektromagnetischen Strahlung und Felder auf dem neuesten Wissensstand der unabhängigen Forschung und der umfangreichen Praxiserfahrungen. Die AefU können entsprechende Hilfen geben (www. aefu.ch);

- Aufklärung der Bevölkerung über mögliche gesundheitliche Risiken strahlungsemittierender Geräte sowie über die Möglichkeit, diese Risiken durch Verzicht oder Ersatz oder Typenwahl und Benutzungsart zu minimieren;

- Information, auch an Schulen, für Kinder und Jugendliche über die sinnvolle und gesundheitlich möglichst risikoarme Nutzung 
1 Steiner-Rüedi E, Gilli Y, Semadeni C, Germann B, Aufdereggen B. Mobilfunk und Gesundheit. Schweiz Ärztezeitung. 2007;88(6):225-8. von Handy, Computer, Internet und elektronischen Unterhaltungsgeräten. Massnahmen zur vorsorglichen Strahlenreduktion sollen thematisiert werden;

- Wahrnehmung der behördlichen Vorbildfunktion durch vorsorglichen Umgang mit NIS-emittierenden Geräten und Installationen;

- Vernetzung aller beteiligten Behördenstellen. Zusammen mit Gesundheitsexpertinnen und Gesundheitsexperten und mit unabhängigen Fachleuten kann erfolgversprechend nach gesundheitsverträglichen Lösungen gesucht werden;

- Bevorzugung emissionsarmer Geräte und Installationen sowie Förderung emissionsarmer Technologien zur Verminderung der Belastung der Bevölkerung mit elektromagnetischer Strahlung;

- initiatives, verantwortungsbewusstes Sicheinschalten in die politische Diskussion über das Thema «Mobilfunk und Gesundheit». Die Gesetz- und Verordnungsgebung des Bundes im Bereich Umwelt- und Gesundheitsschutz sowie Telekommunikation muss auf einer öffentlichen, partizipativen Behandlung dieses Problems basieren. Sie darf keinesfalls der Einflussnahme der Industrie allein überlassen bleiben.

Ärztinnen und Ärzte können in ihrem Umfeld in Praxen und Spitälern und als wichtige Meinungsbildner dazu beitragen, dass EMF vorsorglich reduziert werden. Die AefU bieten Ihnen dazu die nötigen Informationen.

Informationen und die genannten Materialien können elektronisch heruntergeladen werden auf unserer Homepage www.aefu.ch oder sind zu bestellen bei den Ärztinnen und Ärzten für Umweltschutz, Postfach 111, 4013 Basel.

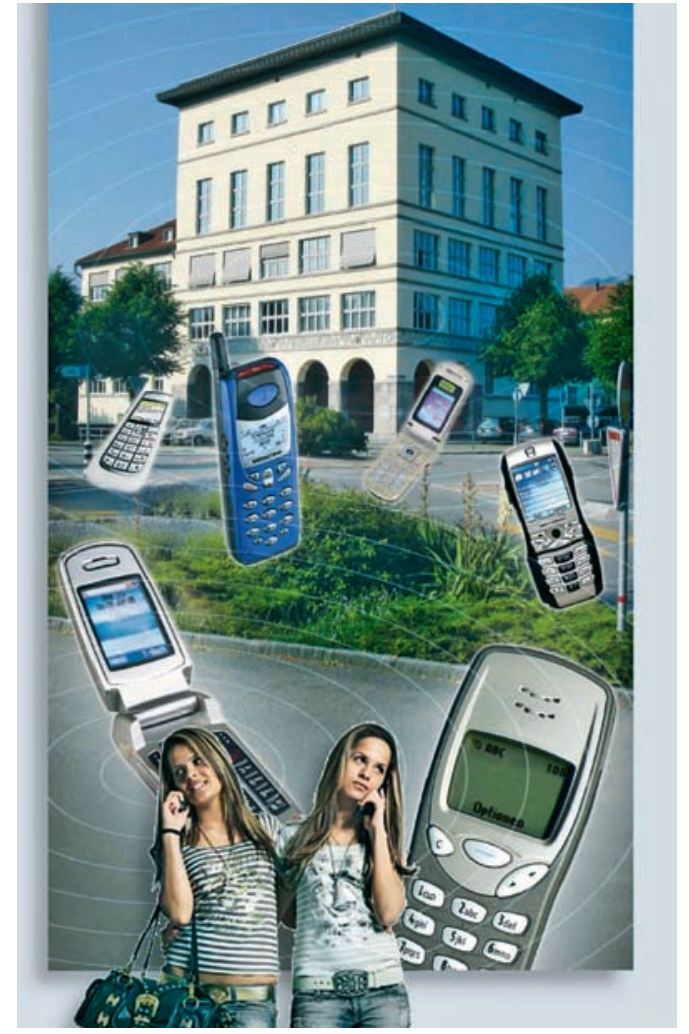

Faltblatt der AefU, AG «Elektromagnetische Felder und Gesundheit» in Zusammenarbeit mit Dr. med. Ludo Cebulla. 